\title{
On Calmness of the Argmin Mapping in Parametric Optimization Problems
}

\author{
Diethard Klatte · Bernd Kummer
}

Received: 18 February 2014 / Accepted: 17 August 2014 / Published online: 9 October 2014

(C) Springer Science+Business Media New York 2014

\begin{abstract}
Recently, Cánovas et al. presented an interesting result: the argmin mapping of a linear semi-infinite program under canonical perturbations is calm if and only if some associated linear semi-infinite inequality system is calm. Using classical tools from parametric optimization, we show that the if-direction of this condition holds in a much more general framework of optimization models, while the opposite direction may fail in the general case. In applications to special classes of problems, we apply a more recent result on the intersection of calm multifunctions.
\end{abstract}

Keywords Calm multifunctions - Parametric optimization problems - Optimal value function · Optimal set mapping · Perturbed nonlinear programs · Convex semi-infinite programs

Mathematics Subject Classification $\quad 49 J 53 \cdot 49 \mathrm{~K} 40 \cdot 90 \mathrm{C} 31 \cdot 90 \mathrm{C} 34$

\section{Introduction}

In this paper, we consider abstract nonlinear optimization problems in a finite dimensional space, where both the objective function and the constraint set depend on some parameter. Given such a problem, Lipschitz properties of the objective function and the

Communicated by Juan Parra.

D. Klatte $(\bowtie)$

IBW, Universität Zürich, Moussonstrasse 15, 8044 Zurich, Switzerland

e-mail: diethard.klatte@business.uzh.ch

B. Kummer

Institut für Mathematik, Humboldt-Universität zu Berlin, Unter den Linden 6, 10099 Berlin, Germany e-mail: kummer@math.hu-berlin.de 
feasible set mapping can be related with Lipschitz properties of the so-called argmin mapping, which assigns to each parameter the (global) optimal solution set. We will focus here to calmness of this multifunction. For the definition of calmness and other Lipschitz-type concepts we refer to Sect. 2, for more details or recent surveys on calmness see, e.g., [1-7].

Our main purpose is twofold: (a) to characterize under suitable assumptions the calmness of the argmin mapping by means of the calmness of an auxiliary mapping, which is defined as some restricted level set mapping, and (b) to apply this to perturbed (standard) finite and semi-infinite nonlinear programs. The stimulus to writing the present note came from a recent paper by Cánovas et al. [1], where such a characterization was given for the special class of linear semi-infinite programs under canonical perturbations. It is shown there that, under the Slater CQ, the argmin mapping is calm if and only if some associated linear semi-infinite inequality system is calm. We will study in how far this can be extended to a larger class of problems. If the constraints are defined by a system of inequalities, then the auxiliary mapping under consideration is given by a system of inequalities, and so the calmness of the argmin mapping can be checked via calmness of a parameter-dependent inequality system. For linear and nonlinear (finite or semi-infinite) inequality systems, there are well-known conditions for calmness, however, sometimes given in equivalent settings like local error bounds or metric subregularity; see, e.g., [4,712].

Note that the authors of [1] essentially used the structure of the linear semi-infinite setting, the special parametrization, and some subdifferential approach to calmness by Azé and Corvellec [13]. In contrast to it, we will apply both classical tools from parametric optimization (cf., e.g., [14-16]) and a basic intersection theorem for calm multifunctions $[3,14]$.

In fact, calmness is a rather weak stability concept for the argmin mapping, since it may happen that, near some solution of the initial problem, there is no solution of the perturbed problem. However, since calmness is a constraint qualification, it can be of value in the study of two-level optimization problems.

The structure of the paper is as follows: In Sect. 2, the basic model and notation are introduced, and some motivation and preliminary results are presented. In Sect. 3, it will be shown that in our abstract setting (cf. (1) below), the calmness of the argmin mapping at some reference point is implied by the calmness of the mentioned auxiliary mapping, provided that the Slater CQ is replaced, e.g., by the Aubin property of the feasible set mapping at the reference point (which is equivalent to the Slater CQ in the framework of [1]). It is worth noting that the proofs do not use any structure of the feasible set and go only back to classical tools in parametric optimization from the 1980ies. Examples will demonstrate that the opposite implication already fails for finite nonlinear programs with linear objective function and a convex quadratic constraint, or with a convex quadratic objective function and linear constraints. In Sect. 4, we will discuss how to verify the assumptions of our main theorem in two standard settings of parametric optimization problems. In particular, we will recall from $[3,14]$ a basic intersection theorem for calm multifunctions and show its application to the special classes of problems under consideration. Section 5 gives some conclusions. 


\section{Notation and Preliminaries}

In this paper, we study the basic parametric optimization problem

$$
P(t): \quad f(x, t) \rightarrow \min _{x} \quad \text { s.t. } \quad x \in M(t), t \text { varies near } \bar{t},
$$

where we assume throughout that $T$ is a normed linear space, the multifunction $M$ : $T \rightrightarrows \mathbb{R}^{n}$ is closed (i.e., its graph, denoted by gph $M$, is closed), a reference point $(\bar{t}, \bar{x}) \in \operatorname{gph} M$ is given, and the function $f: \mathbb{R}^{n} \times T \rightarrow \mathbb{R}$ is Lipschitzian in some neighborhood of $(\bar{t}, \bar{x})$. Recall that the domain and the graph of a multifunction $\Phi: T \rightrightarrows \mathbb{R}^{n}$ are defined by dom $\Phi:=\{t \in T \mid \Phi(t) \neq \emptyset\}$ and $\operatorname{gph} \Phi:=\{(t, x) \in$ $\left.T \times \mathbb{R}^{n} \mid x \in \Phi(t)\right\}$, respectively.

$M$ will be called the feasible set mapping of (1). Define by

$$
\begin{aligned}
\Psi(t) & :=\operatorname{argmin}_{x}\{f(x, t) \mid x \in M(t)\}, \quad t \in T, \\
\varphi(t) & :=\inf _{x}\{f(x, t) \mid x \in M(t)\}, \quad t \in T,
\end{aligned}
$$

the argmin mapping (or optimal solution set mapping) and the infimum value function, respectively, of the parametric program (1).

Moreover, we define two auxiliary multifunctions,

$$
\begin{aligned}
\Psi^{0}(t) & :=\operatorname{argmin}_{x}\{f(x, \bar{t}) \mid x \in M(t)\}, \quad t \in T, \\
L(t, \mu) & :=\{x \in M(t) \mid f(x, \bar{t}) \leq \mu\}, \quad t \in T, \mu \in \mathbb{R} .
\end{aligned}
$$

Our aim is to characterize, under certain assumptions, the calmness of the argmin mappings $\Psi$ and $\Psi^{0}$ by means of the calmness of the auxiliary mapping $L$, and to apply this to perturbed (finite and semi-infinite) nonlinear programs. We start with the stability notions needed in the following. Denote by $B$ the closed unit ball in $T$ or $\mathbb{R}^{n}$ and by $B(z, r)$ the closed $r$-neighborhood of $z$ in $T$ or $\mathbb{R}^{n}$ (in the corresponding norms). We use the symbol $\|\cdot\|$ both for the norms in $\mathbb{R}^{n}$ and $T$ and put $\|(x, t)\|:=\|x\|+\|t\|$. Further, write $\operatorname{dist}(z, X):=\inf _{x \in X}\|z-x\|$ (with $\left.\operatorname{dist}(z, \emptyset):=+\infty\right)$ for the distance of $z \in \mathbb{R}^{n}$ to $X$, and let $X+r Y:=\{x+r y \mid x \in X, y \in Y\}$ for $X, Y \subset \mathbb{R}^{n}$ and $r \in \mathbb{R}$.

Let a multifunction $\Phi: T \rightrightarrows \mathbb{R}^{n}$ and $(\bar{t}, \bar{x}) \in \operatorname{gph} \Phi$ be given. $\Phi$ is called calm at $(\bar{t}, \bar{x})$ iff there are $\varepsilon, \delta, \varrho>0$ such that

$$
\Phi(t) \cap B(\bar{x}, \varepsilon) \subset \Phi(\bar{t})+\varrho\|t-\bar{t}\| B, \forall t \in B(\bar{t}, \delta),
$$

where $\Phi(t) \cap B(\bar{x}, \varepsilon)=\emptyset$ for $t \neq \bar{t}$ is possible. $\Phi$ is said to have the Aubin property at $(\bar{t}, \bar{x})$ iff there are $\varepsilon, \delta, \varrho>0$ such that

$$
\Phi(t) \cap B(\bar{x}, \varepsilon) \subset \Phi\left(t^{\prime}\right)+\varrho\left\|t-t^{\prime}\right\| B, \forall t, t^{\prime} \in B(\bar{t}, \delta) .
$$

$\Phi$ is called Lipschitz lower semicontinuous (Lipschitz l.s.c.) at $(\bar{t}, \bar{x})$ iff there are $\delta, \varrho>0$ such that

$$
\operatorname{dist}(\bar{x}, \Phi(t)) \leq \varrho\|t-\bar{t}\|, \forall t \in B(\bar{t}, \delta) .
$$


Note that (6) implies for each $\varepsilon>0, \Phi(t) \cap B(\bar{x}, \varepsilon) \neq \emptyset$ if $\|t-\bar{t}\| \leq \min \{\delta, \varepsilon / \varrho\}$. Obviously, the Aubin property implies both calmness and Lipschitz lower semicontinuity. Note that the opposite direction fails, let, e.g., $\Phi: \mathbb{R} \rightrightarrows \mathbb{R}$ with $\Phi(t)=\{0\}$ for $t \neq 0, \Phi(0)=\mathbb{R}$, and $(\bar{t}, \bar{x})=(0,0)$.

Now we recall the mentioned result by Cánovas et al. [1], which gave the impetus for our studies and has been devoted to the following special setting of problem (1) with parameter space $T=\mathbb{R}^{n} \times C(I, \mathbb{R})$ :

$$
t=(c, b) \in T, f(x, t)=c^{\prime} x, M(t)=\left\{x \in \mathbb{R}^{n} \mid a_{i}^{\prime} x \leq b_{i}, i \in I\right\},
$$

where $I$ is a compact Hausdorff space, $C(I, \mathbb{R})$ is the linear space of continuous functions $i \in I \mapsto b_{i}$ equipped with the norm $\|b\|:=\max _{i \in I}\left|b_{i}\right|$, and $a \in(C(I, \mathbb{R}))^{n}$ is given. This is a linear semi-infinite program with canonical perturbations $t=(c, b)$.

The main statement in [1, Thm. 3.1, Rem. 3.1] in the setting (7) says

Proposition 2.1 For a reference point $(\bar{t}, \bar{x}) \in \operatorname{gph} \Psi$ with $\bar{t}=(\bar{c}, \bar{b})$ and under the Slater $C Q$ at $\bar{b}$ (i.e., for some $\tilde{x}$, it holds $a_{i}^{\prime} \tilde{x}<\bar{b}_{i}, \forall i \in I$ ), the following properties are pairwise equivalent:

(i) $\Psi$ is calm at $(\bar{t}, \bar{x})$,

(ii) $\Psi^{0}$ is calm at $(\bar{b}, \bar{x})$,

(iii) $L$ is calm at $\left(\left(\bar{t}, \bar{c}^{\prime} \bar{x}\right), \bar{x}\right)$.

Hence, in particular, the calmness of the $\operatorname{argmin}$ mapping $\Psi=\Psi(c, b)$ can be checked by the calmness of $L=L(b, \mu)$, which is described by an inequality system with right-hand side perturbations only.

\section{Calmness Conditions for the Argmin Mapping}

In this section, we derive the main result of our note, namely, the implication $L \mathrm{calm}$ $\Rightarrow \Psi$ calm for the general model. Consider again the basic parametric optimization problem (1) and assume that

$M$ is a closed multifunction, $(\bar{t}, \bar{x}) \in \operatorname{gph} \Psi$ is a given point, and $f$ is Lipschitzian on some neighborhood $\Omega_{f}$ of $(\bar{x}, \bar{t})$ with modulus $\varrho_{f}>0$.

There are some standard tools in parametric optimization, which relate Lipschitz properties of the objective function $f$ and the feasible set mapping $M$ to a Lipschitz property of the optimal value function. Define the mappings

$$
\begin{aligned}
& \Psi_{V}(t):=\operatorname{argmin}_{x}\{f(x, t) \mid x \in M(t) \cap V\}, \quad t \in T, \\
& \varphi_{V}(t):=\inf _{x}\{f(x, t) \mid x \in M(t) \cap V\}, \quad t \in T,
\end{aligned}
$$

for given $V \subset \mathbb{R}^{n}$. Following [6], we will use the notion of calmness of a function and say that $\varphi_{V}$ is calm at $\bar{t} \in \operatorname{dom} \varphi_{V}$ (also called pointwise Lipschitz at $\bar{t}$ ) iff there is some neighborhood $D$ of $\bar{t}$ such that

$$
\left|\varphi_{V}(t)-\varphi_{V}(\bar{t})\right| \leq \varrho\|t-\bar{t}\| \text { holds for some } \varrho \geq 0 \text { and all } t \in \operatorname{dom} \varphi_{V} \cap D \text {. }
$$


We borrow here an idea from $[15,16]$ to get the following result.

Lemma 3.1 Consider the problem (1) under the assumptions (8). Suppose for some $\varepsilon, \delta, \varrho_{M}>0$ and $U=B(\bar{t}, \delta), V=B(\bar{x}, \varepsilon)$ that one has $V \times U \subset \Omega_{f}$ and

$$
\begin{aligned}
\emptyset \neq M(t) \cap V & \subset M(\bar{t})+\varrho_{M}\|t-\bar{t}\| B, \forall t \in U, \\
\operatorname{dist}(\bar{x}, M(t)) & \leq \varrho_{M}\|t-\bar{t}\|, \forall t \in U .
\end{aligned}
$$

Then, the function $\varphi_{V}$ is calm at $\bar{t}$.

Proof Let $\delta^{\prime}=\varepsilon / \varrho_{M}$ and $t \in U \cap B\left(\bar{t}, \delta^{\prime}\right)$. Then, $M(t) \cap V$ is nonempty and compact, and hence, by continuity of $f$ and the Weierstrass Theorem, we observe $\Psi_{V}(t) \neq \varnothing$. By (10), we then find for any $y \in \Psi_{V}(t)$ some $\bar{y} \in M(\bar{t})$ such that $\|y-\bar{y}\| \leq \varrho_{M}\|t-\bar{t}\| \leq \varepsilon$. Hence, Lipschitz continuity of $f$ on $V \times U$ leads to

$\varphi_{V}(\bar{t}) \leq f(\bar{y}, \bar{t}) \leq f(y, t)+|f(y, t)-f(\bar{y}, \bar{t})| \leq \varphi_{V}(t)+\varrho_{f}(\|y-\bar{y}\|+\|t-\bar{t}\|)$,

and so

$$
\varphi_{V}(\bar{t}) \leq \varphi_{V}(t)+\varrho_{f}\left(\varrho_{M}+1\right)\|t-\bar{t}\|
$$

Furthermore, by (11) there is some $z \in M(t)$ such that $\|z-\bar{x}\| \leq \varrho_{M}\|t-\bar{t}\| \leq \varepsilon$, and it follows by similar arguments

$$
\varphi_{V}(t) \leq f(z, t) \leq f(\bar{x}, \bar{t})+|f(z, t)-f(\bar{x}, \bar{t})| \leq \varphi_{V}(\bar{t})+\varrho_{f}\left(\varrho_{M}+1\right)\|t-\bar{t}\| .
$$

Therefore, $\varphi_{V}$ is calm at $\bar{t}$ with modulus $\varrho_{f}\left(\varrho_{M}+1\right)$.

Now we relate Lipschitz properties of the objective function and the feasible set mapping with the calmness of the argmin mapping under the assumption that the auxiliary multifunction $L$, introduced in (3), is calm.

Theorem 3.1 Consider the problem (1) under the assumptions (8). Suppose that, for the reference point $(\bar{t}, \bar{x}) \in \operatorname{gph} \Psi$,

(i) the feasible set mapping $M$ is calm and Lipschitz l.s.c. at $(\bar{t}, \bar{x})$ and

(ii) the multifunction $L=L(t, \mu)$ in (3) is calm at $((\bar{t}, \varphi(\bar{t})), \bar{x})$.

Then, the argmin mapping $\Psi$ is calm at $(\bar{t}, \bar{x})$.

Proof Let us start with the simple observation that, for given $t \in T$ and $V \subset \mathbb{R}^{n}$,

$$
\Psi(t) \cap V \neq \emptyset \Rightarrow \Psi_{V}(t)=\Psi(t) \cap V
$$

Indeed, if $\Psi(t) \cap V \neq \emptyset$, then $\varphi(t)=f\left(x_{t}, t\right) \leq f(x)$ particularly holds for some $x_{t} \in M(t) \cap V$ and all $x \in M(t) \cap V$. Hence, $\varphi(t)=\varphi_{V}(t)$ and so

$$
\Psi_{V}(t)=M(t) \cap V \cap\{x \mid f(x, t)=\varphi(t)\}=\Psi(t) \cap V,
$$


which gives (12), and we turn to the main part of the proof.

Since $M$ and $L$ are calm at $(\bar{t}, \bar{x})$ and $((\bar{t}, \varphi(\bar{t})), \bar{x})$, respectively, we may assume that there are some positive real numbers $\delta, \varepsilon, \varrho_{M}$, and $\varrho_{L}$ such that with $U=B(\bar{t}, \delta)$, $V=B(\bar{x}, \varepsilon)$ and $\bar{\mu}=\varphi(\bar{t})$, both the relations

$$
\begin{aligned}
M(t) & \cap V \subset M(\bar{t})+\varrho_{M}\|t-\bar{t}\| B, \forall t \in U, \quad \text { and } \\
L(t, \mu) & \cap V \subset L(\bar{t}, \bar{\mu})+\varrho_{L}(\|t-\bar{t}\|+|\mu-\bar{\mu}|) B, \quad \forall t \in U, \forall \mu \in B(\bar{\mu}, \delta),
\end{aligned}
$$

hold true. Let $U$ and $V$ be small enough such that $f$ is Lipschitzian on $V \times U$ with modulus $\varrho_{f}$ according to (8). Since $M$ is Lipschitz l.s.c. at $(\bar{t}, \bar{x})$, the radius $\delta>0$ may be considered already small enough such that (11) and

$$
M(t) \cap V \neq \emptyset, \forall t \in U=B(\bar{t}, \delta)
$$

are satisfied. Hence, we may assume the relations (10), (11) and (13) hold all together. By Lemma 3.1, then there is some modulus $\varrho_{\varphi}>0$ such that, for some neighborhood $\tilde{U} \subset U$ of $\bar{t}$,

$$
\left|\varphi_{V}(t)-\varphi_{V}(\bar{t})\right| \leq \varrho_{\varphi}\|t-\bar{t}\|, \forall t \in \tilde{U} \cap \operatorname{dom} \Psi_{V}
$$

Now let $U^{\prime} \subset \tilde{U}$ be a (closed) neighborhood of $\bar{t}$ such that, for all $t \in U^{\prime}$ and all $x \in V$, both

$$
\varrho_{\varphi}\|t-\bar{t}\| \leq \frac{\delta}{2} \text { and }|f(x, t)-f(x, \bar{t})| \leq \varrho_{f}\|t-\bar{t}\| \leq \frac{\delta}{2}
$$

hold true. By definition and (12), one has $L(\bar{t}, \varphi(\bar{t}))=\Psi(\bar{t}), \Psi_{V}(\bar{t})=\Psi(\bar{t}) \cap V$ and

$$
\begin{aligned}
x \in \Psi(t) & \Leftrightarrow(x \in M(t) \text { and } f(x, t) \leq \varphi(t)) \\
& \Leftrightarrow(x \in M(t) \text { and } f(x, \bar{t}) \leq \varphi(t)+f(x, \bar{t})-f(x, t)) \\
& \Leftrightarrow x \in L(t, \mu(x, t)) \text { where } \mu(x, t):=\varphi(t)+f(x, \bar{t})-f(x, t) .
\end{aligned}
$$

Consider any $t \in U^{\prime}$ and suppose $\Psi(t) \cap V \neq \emptyset$, otherwise the calmness definition with respect to $U^{\prime}$ and $V$ is trivially satisfied. Hence, we obtain due to (12)

$$
\Psi_{V}(t)=\Psi(t) \cap V \text { and } \varphi(t)=\varphi_{V}(t)
$$

as well as, by (14) and (15),

$$
\begin{aligned}
|\mu(x, t)-\varphi(\bar{t})| & =\left|\varphi_{V}(t)+f(x, \bar{t})-f(x, t)-\varphi_{V}(\bar{t})\right| \\
& \leq\left|\varphi_{V}(t)-\varphi_{V}(\bar{t})\right|+|f(x, \bar{t})-f(x, t)| \leq \delta .
\end{aligned}
$$

This allows to apply (13) (recall $\bar{\mu}=\varphi(\bar{t})$ and $L(\bar{t}, \bar{\mu})=\Psi(\bar{t})$ ), and it follows

$$
\Psi(t) \cap V=L(t, \mu(x, t)) \cap V \subset \Psi(\bar{t})+\varrho_{L}(\|t-\bar{t}\|+|\mu(x, t)-\bar{\mu}|) B
$$


where $|\mu(x, t)-\bar{\mu}| \leq\left|\varphi_{V}(t)-\varphi_{V}(\bar{t})\right|+|f(x, \bar{t})-f(x, t)| \leq\left(\varrho_{\varphi}+\varrho_{f}\right)\|t-\bar{t}\|$, i.e.,

$$
\left.\Psi(t) \cap V \subset \Psi(\bar{t})+\varrho_{L}\left(1+\varrho_{\varphi}+\varrho_{f}\right)\|t-\bar{t}\|\right) B
$$

This completes the proof.

In consequence, we have at the related points that

$$
L \text { is calm } \Rightarrow \Psi \text { is calm (and hence, trivially, } \Psi^{0} \text { is calm), }
$$

provided $M$ is calm and Lipschitz lower semicontinuous. The opposite implication is not true; this is illustrated by the following simple examples. Both examples concern the model (7) with canonical perturbations, except for the linearity of all problem functions.

Example 3.1 Consider the parametric problem

$$
\min x^{2}-c x \text { s.t. }-1+b_{1} \leq x \leq 1+b_{2}, \quad\left(c, b_{1}, b_{2}\right) \text { varies near }(0,0,0) .
$$

Obviously, $\Psi(c, b)=\left\{\frac{1}{2} c\right\}$ for small $|c|$ and $b=\left(b_{1}, b_{2}\right)$ near 0 . Hence, $\Psi$ is calm at the origin, the same for $\Psi^{0}(b)=\Psi(0, b)$, while $\varphi(c, b)=-\frac{1}{4} c^{2}$ is Lipschitz near the origin. The Slater CQ is satisfied; hence the constraint set mapping $M$ has the Aubin property. For $\mu=\varepsilon^{2}(\varepsilon>0$ small $)$ and $b=0$, the set

$$
L(0, \mu)=\left\{x \mid-1 \leq x \leq 1, x^{2} \leq \mu\right\}
$$

contains $x_{\mu}=\varepsilon$. Since $\operatorname{dist}\left(x_{\mu}, L(0,0)\right)=\varepsilon=\sqrt{\mu}$, the mapping $L$ is not calm at the origin. In the example, the level sets of the objective function, $F(\mu)=\left\{x \mid x^{2} \leq \mu\right\}$, are not calm at $(\bar{\mu}, \bar{x})=(0,0)$.

Example 3.2 For the canonically perturbed problem

$$
\min y-c_{1} x-c_{2} y \quad \text { s.t. } x^{2}-y \leq b, \quad\left(c_{1}, c_{2}, b\right) \text { varies near }(0,0,0),
$$

the optimal solution mapping

$$
\Psi\left(c_{1}, c_{2}, b\right)=\left\{\left(\frac{c_{1}}{2\left(1-c_{2}\right)}, \frac{c_{1}^{2}}{4\left(1-c_{2}\right)^{2}}-b\right)\right\}
$$

is Lipschitz near $(0,0,0)$, and hence calm at the origin, the same for the mapping $\Psi^{0}(b)=\Psi(0,0, b)$. However,

$$
L(b, \mu)=\left\{(x, y) \mid y \leq \mu, x^{2}-y \leq b\right\}
$$

is not calm at the origin, one has only to choose $b \equiv 0$ and $\mu \downarrow 0$. Again, the Slater CQ is satisfied, and so the constraint set mapping $M$ has the Aubin property. In contrast to 
Example 3.1, the level set mapping of the objective function, $F(\mu)=\{(x, y) \mid y \leq \mu\}$, is calm at the origin.

\section{Calm Intersections and Application to Special Classes}

In this section, the assumptions of Theorem 3.1 are discussed in the context of two special classes of optimization problems (1) including canonical perturbations: (i) a perturbed (finite) nonlinear optimization problem with differentiable data, (ii) a perturbed convex semi-infinite optimization problem covering the model studied in [1].

Of particular interest are calmness conditions for the intersection mapping $L(t, \mu)=M(t) \cap\{x \mid f(x, \bar{t}) \leq \mu\}$. For this reason, we start by recalling from $[3,14]$ some basic intersection theorem for calm multifunctions.

\subsection{Calm Intersections}

Below, we shall apply Thm. 2.5 in [3] (cf. also [14, Thm. 3.6]) for closed mappings $S: Y \rightrightarrows X$ and $T: Z \rightrightarrows X$ between metric spaces $X, Y, Z$, namely,

Theorem 4.1 (calm intersections) Let $S$ be calm at $(\bar{y}, \bar{x}), T$ be calm at $(\bar{z}, \bar{x})$ and $T^{-1}$ be pseudo-Lipschitz (i.e., have the Aubin property) at $(\bar{x}, \bar{z})$. Moreover, let $H(z)=$ $S(\bar{y}) \cap T(z)$ be calm at $(\bar{z}, \bar{x})$. Then, $\Sigma(y, z)=S(y) \cap T(z)$ is calm at $(\bar{y}, \bar{z}, \bar{x})$.

Note. Of course, if $\Sigma$ is calm, then the restricted mapping $H$ is also calm at the related point.

Example 4.1 To illustrate the theorem, consider $\Sigma(y, z)=S(y) \cap T(z)$, where $y=$ $\left(y_{1}, y_{2}\right) \in \mathbb{R}^{2}, z \in \mathbb{R}$,

$$
S(y)=\left\{\left(x_{1}, x_{2}\right) \mid x_{2}+x_{1}^{2} \geq y_{1}, x_{2} \geq y_{2}\right\}, T(z)=\left\{\left(x_{1}, x_{2}\right) \mid x_{2} \leq z\right\} .
$$

Put $\bar{x}_{1}=\bar{x}_{2}=\bar{y}_{1}=\bar{y}_{2}=\bar{z}=0$. Then, the Mangasarian-Fromovitz constraint qualification $(M F C Q)$ is satisfied for $S(0)$ at $\bar{x}=0$. Therefore, by Robinson's classical result [17], $S$ is even pseudo-Lipschitz and hence calm at the origin. $T$ and $T^{-1}$ (given by linear inequalities) are calm and pseudo-Lipschitz.

Finally, we consider $H(z)=S(0) \cap T(z)$. If $z<0$, then $H(z)=\emptyset$, while for $z \geq 0, H(z)$ is given by the linear inequalities $0 \leq x_{2} \leq z$, since $x_{2} \geq 0$ implies $x_{2}+x_{1}^{2} \geq 0$. In consequence, $H$ is calm at the origin, and the theorem says that $\Sigma$ has the same property.

When applying the theorem to the model (1) and the definition (3) of $L$, with $f(x)=$ $f(x, \bar{t})$ for some given $\bar{t}$, we obtain

$$
L(t, \mu)=F(\mu) \cap M(t), \quad \text { where } F(\mu)=\{x \mid f(x) \leq \mu\}
$$

In this context, we may put $S=F, T=M$ in order to obtain 
Proposition 4.1 Let $F$ be calm at $(\bar{\mu}, \bar{x}), M$ be calm at $(\bar{t}, \bar{x})$ and $M^{-1}$ have the Aubin property at $(\bar{x}, \bar{t})$. Moreover, let $H_{1}(t)=F(\bar{\mu}) \cap M(t)$ be calm at $(\bar{t}, \bar{x})$. Then, $L$ is calm at $(\bar{t}, \bar{\mu}, \bar{x})$.

Setting $S=M, T=F$ we obtain

Proposition 4.2 Let $M$ be calm at $(\bar{t}, \bar{x}), F$ be calm at $(\bar{\mu}, \bar{x})$ and $F^{-1}$ have the Aubin property at $(\bar{x}, \bar{\mu})$. Moreover, let $H_{2}(\mu)=M(\bar{t}) \cap F(\mu)$ be calm at $(\bar{\mu}, \bar{x})$. Then, $L$ is calm at $(\bar{t}, \bar{\mu}, \bar{x})$.

Again, calmness of $H_{1}$ and $H_{2}$, respectively, is necessary for calmness of $L$.

Example 4.2 Using example 4.1 we may easily obtain calm mappings $L$ and $\Psi^{0}$ at the origin. Consider (the non-convex problem)

$$
P\left(t_{1}, t_{2}\right): \quad \min \left\{x_{2} \mid x_{2}+x_{1}^{2} \geq t_{1}, x_{2} \geq t_{2}\right\} .
$$

Putting $\mu=z$ in $(16), L(\bar{t}, \mu)=L(0, \mu)$ coincides with $H(z)$ of Example 4.1. Moreover, we have

$$
\Psi^{0}(0,0)=\left\{\left(x_{1}, 0\right) \mid x_{1} \in \mathbb{R}\right\}, \Psi^{0}\left(t_{1}, t_{2}\right)=\left\{\left(x_{1}, t_{2}\right) \mid t_{2}+x_{1}^{2} \geq t_{1}\right\}
$$

If $t_{2} \geq t_{1}$, again all components $x_{1}$ are allowed in $\Psi^{0}\left(t_{1}, t_{2}\right)$. If $t_{2}<t_{1}$, then $x_{1}^{2} \geq t_{1}-t_{2}$ is required, but $\operatorname{dist}\left(\left(x_{1}, t_{2}\right), \Psi^{0}(0,0)\right) \leq\left|t_{2}\right|$ verifies calmness of $\Psi^{0}$.

Replacing $x_{1}^{2}$ by $-x_{1}^{2}$ one obtains a well-known non-calm example satisfying the Slater CQ.

Note that, for the calmness assumption on the level set mapping $F$, there are several known conditions how to check this; see, e.g., $[4,9,10,13,18,19]$. It is automatically satisfied if $f(\cdot, \bar{t})$ is linear-affine.

Evidently, $F^{-1}(x)=\{\mu \mid \mu \geq f(x)\}$ has the Aubin property, if $f$ is locally Lipschitz. Similarly, $M^{-1}(x)=\{b \mid b \geq g(x)\}$ has the Aubin property, if $g: \mathbb{R}^{n} \rightarrow$ $\mathbb{R}^{m}$ is locally Lipschitz. All these mappings are calm for usual linear programming (cf. [8]).

In Example 3.2 ( $L$ is not calm) all assumptions of Prop. 4.2 are satisfied, except for the calmness of the mapping

$$
H_{2}(\mu)=M(0) \cap F(\mu)=\left\{(x, y) \mid x^{2} \leq y, y \leq \mu\right\}
$$

while in Example 3.1 both $F$ and $H_{2}$ are not calm.

4.2 Nonlinear Programs with Differentiable Data

Consider the parametric optimization problem

$$
P(t), t=(p, c, b): \quad h(x, p)+c^{\prime} x \rightarrow \min _{x} \quad \text { s.t. } \quad g_{i}(x, p) \leq b_{i}, i \in I,
$$


where $I=\{1, \ldots, m\}, t \in T=\mathbb{R}^{q+n+m}$ varies near some reference parameter $\bar{t}=$ $(\bar{p}, \bar{c}, \bar{b})$, and the functions $h, g_{i}$ are continuously differentiable on some neighborhood of a given pair $(\bar{x}, \bar{p})$. The canonical perturbations $(c, b)$ were included to obtain equivalent conditions for the assumed Lipschitz stability properties. It is not a problem to add finitely many equality constraints, but we avoid this to keep technicalities as small as possible. Define

$$
\begin{aligned}
f(x, t) & =f(x, p, c):=h(x, p)+c^{\prime} x, t \in T, \\
M(t) & =M(p, b):=\left\{x \in \mathbb{R}^{n} \mid g_{i}(x, p) \leq b_{i}, i \in I\right\}, \quad t \in T
\end{aligned}
$$

then the multifunctions $\Psi, \Psi^{0}, L$, and $F$ are defined as above. Suppose that $(\bar{t}, \bar{x}) \in$ gph $\Psi$ with $\bar{t}=(\bar{p}, \bar{c}, \bar{b})$, and let us discuss the assumptions of Theorem 3.1.

$M$ is calm and Lipschitz l.s.c.: $M$ is Lipschitz 1.s.c. at $((\bar{p}, \bar{b}), \bar{x})$ if and only if the MFCQ is satisfied for $M(\bar{p}, \bar{b})$ at $\bar{x}$; see [11, Lemma 1]. This, however, is by Robinson's classical result [17] equivalent to the Aubin property of $M$ at $((\bar{p}, \bar{b}), \bar{x})$, which implies calmness of $M$ at this point.

$L$ is calm: Let us discuss the assumptions of Prop. 4.2. Because of the previous observation we assume MFCQ at the point of interest. Hence $M$ is calm. Since the data are $C^{1}$ and hence locally Lipschitz, $F^{-1}$ has the Aubin property-as discussed above.

Let $\bar{\mu}=f(\bar{x}, \bar{t})$, and define $q(x):=f(x, \bar{t})-\bar{\mu}, d:=\operatorname{dim}\left[\nabla q(\bar{x}) \mathbb{R}^{n}\right]$ and $d_{\varepsilon}:=\operatorname{dim}\left[q\left(B(\bar{x}, \varepsilon) \cap \mathbb{R}^{+}\right]\right.$for $\varepsilon>0$. Then, $F(\beta)=\{x \mid q(x) \leq \beta\}$ is calm at $(0, \bar{x})$ if and only if there is some $\varepsilon_{0}>0$ such that $d=d_{\varepsilon}$ for all $\varepsilon \in\left(0, \varepsilon_{0}\right)$ (cf. [19, Prop. 3.13]). Equivalently, $F$ is calm at $(0, \bar{x})$ if and only if either $\nabla q(\bar{x}) \neq 0$ holds true, or $\bar{x}$ is a local maximizer of $q$ (cf. [4, Prop. 3]).

It remains to check that the mapping

$$
H_{2}(\mu)=M(\bar{t}) \cap F(\mu)=\left\{x \mid g(x, \bar{p}) \leq \bar{b}, h(\bar{p}, x)+\bar{c}^{\prime} x \leq \mu\right\}
$$

is calm. Obviously, $H_{2}(\mu)$ is now defined by a finite inequality system with differentiable data and right-hand side perturbations, and one can apply corresponding calmness characterizations for such systems; see, e.g., [4,7,9-12].

\subsection{Convex Semi-infinite Optimization Problems}

Here, the basic model is the canonically perturbed semi-infinite program

$$
P(t), t=(c, b): \quad h(x)+c^{\prime} x \rightarrow \min _{x} \quad \text { s.t. } \quad g_{i}(x) \leq b_{i}, i \in I,
$$

where we suppose throughout that the index set $I$ is a compact Hausdorff space, the real-valued functions $h, g_{i}(i \in I)$ are convex on $\mathbb{R}^{n},(i, x) \mapsto g_{i}(x)$ is continuous, the pair $(c, b)$ varies in the parameter space $T:=\mathbb{R}^{n} \times C(I, \mathbb{R})$ near some given $\bar{t}=(\bar{c}, \bar{b}) \in T$. The multifunctions $\Psi, \Psi^{0}, L$, and $F$ are defined as above, when 
setting

$$
\begin{aligned}
f(x, t) & =f(x, c):=h(x)+c^{\prime} x, \quad t \in T, \\
M(t) & =M(b):=\left\{x \in \mathbb{R}^{n} \mid g_{i}(x) \leq b_{i}, \quad i \in I\right\}, \quad t \in T .
\end{aligned}
$$

The model (18) covers the linear semi-infinite setting in (7), discussed in [1], by putting $h(x) \equiv 0$ and $g_{i}(x)=a_{i}^{\prime} x$. In [1], the Slater constraint qualification (SCQ) was supposed in the equivalence theorem, which was recalled in Prop. 2.1 above. Let us also suppose SCQ at $M(\bar{b})$, i.e.,

$$
\exists \widetilde{x}: \quad g_{i}(\widetilde{x})<\bar{b}_{i}, \forall i \in I .
$$

We again check the assumptions of Theorem 3.1. Suppose $(\bar{t}, \bar{x}) \in \operatorname{gph} \Psi$.

$M$ is calm and Lipschitz l.s.c.: SCQ at $M(\bar{b})$ implies that $M$ has the Aubin property at $(\bar{b}, \bar{x})$, and vice versa. This equivalence is essentially a consequence of the RobinsonUrsescu Theorem (cf. [20]). It was proved for the linear semi-infinite setting in [21, Thm. 2.1], for $I$ being a compact Hausdorff space, and in our setting (18) in [22, Lemma 3], for $I$ being a compact metric space. Hence, under SCQ at $M(\bar{b}), M$ is both calm and Lipschitz 1.s.c at $(\bar{b}, \bar{x})$.

$L$ is calm: Again, we discuss the assumptions of Prop. 4.2. $M$ is calm because of the SCQ. $F^{-1}$ has the Aubin property, since $f$ is convex.

For calmness of the convex level set mapping $F$ see, e.g., $[4,5,9,10,18]$. In particular, if $\bar{x}$ is not an unconstrained minimizer of $f(\cdot, \bar{c})$, then $F$ is calm at $(\bar{\mu}, \bar{x})$, since the Slater condition for the level set mapping $F$ holds, i.e., $f(\tilde{x}, \bar{c})<f(\bar{x}, \bar{c})$ for some $\tilde{x}$. This is equivalent to the Aubin property of $F$ at $(\bar{\mu}, \bar{x})$ with $\bar{\mu}=f(\bar{x}, \bar{c})$, by the Robinson-Ursescu theorem [20].

It remains to check that

$$
H_{2}(\mu)=M(\bar{b}) \cap\left\{x \mid h(x)+\bar{c}^{\prime} x \leq \mu\right\}
$$

is calm at $(\bar{\mu}, \bar{x})$, but this reduces to calmness of a (semi-infinite) inequality system with right-hand side perturbations, for this one finds conditions, e.g., in $[1,4,9,11]$.

\section{Conclusions}

This paper has been devoted to the question whether calmness of the optimal set mapping of a parameter-dependent nonlinear program at some reference point can be characterized by calmness of some restricted level set mapping, provided the feasible set mapping is calm and Lipschitz l.s.c. at the given point. As mentioned in the introduction, this question was inspired by a recent positive answer to it in the context of canonically perturbed linear semi-infinite problems, given by Canovas et al. [1] (see Proposition 2.1 above). It has turned out that one direction of this equivalence can be extended to a wide class of parametric nonlinear programs, in particular to perturbed (finite) nonlinear programs with differentiable data and canonically perturbed convex semi-infinite programs: calmness of the restricted level set mapping $L$ defined in 
Sect. 2 implies calmness of the optimal set mapping under some CQ. Simple examples have shown that the opposite direction is not true if the objective function or some constraints are nonlinear.

Acknowledgments The authors would like to thank the two referees and the associate editor for their constructive comments.

\section{References}

1. Cánovas, M.J., Hantoute, A., Parra, J., Toledo, F.J.: Calmness of the argmin mapping in linear semiinfinite optimization. J. Optim. Theory Appl. 160, 111-126 (2014)

2. Rockafellar, R.T., Wets, R.J.-B.: Variational Analysis. Springer, Berlin (1998)

3. Klatte, D., Kummer, B.: Nonsmooth Equations in Optimization-Regularity, Calculus, Methods and Applications. Kluwer Academic Publ., Dordrecht (2002)

4. Henrion, R., Outrata, J.: Calmness of constraint systems with applications. Math. Program. B 104, 437-464 (2005)

5. Ioffe, A.D., Outrata, J.: On metric and calmness qualification conditions in subdifferential calculus. Set Valued Anal. 16, 199-227 (2008)

6. Dontchev, A.L., Rockafellar, R.T.: Implicit Functions and Solution Mappings. Springer, New York (2009)

7. Gfrerer, H.: First order and second order characterizations of metric subregularity and calmness of constraint set mappings. SIAM J. Optim. 21, 1439-1474 (2011)

8. Robinson, S.M.: Some continuity properties of polyhedral multifunctions. Math. Program. Study 14, 206-214 (1981)

9. Li, W.: Abadie's constraint qualification, metric regularity, and error bounds for differentiable convex inequalities. SIAM J. Optim. 7, 966-978 (1997)

10. Pang, J.S.: Error bounds in mathematical programming. Math. Program. 79, 299-332 (1997)

11. Klatte, D., Kummer, B.: Optimization methods and stability of inclusions in Banach spaces. Math. Program. B 117, 305-330 (2009)

12. Kummer, B.: Inclusions in general spaces: Hoelder stability, solution schemes and Ekeland's principle. J. Math. Anal. Appl. 358, 327-344 (2009)

13. Azé, D., Corvellec, J.-N.: Characterizations of error bounds for lower semicontinuous functions on metric spaces. ESIAM Control Optim. Calc. Var. 10, 409-425 (2004)

14. Klatte, D., Kummer, B.: Constrained minima and Lipschitzian penalties in metric spaces. SIAM J. Optim. 13, 619-633 (2002)

15. Alt, W.: Lipschitzian perturbations of infinite optimization problems. In: Fiacco, A.V. (ed.) Mathematical Programming with Data Perturbations, pp. 7-21. M. Dekker, New York (1983)

16. Klatte, D.: On the Stability of Local and Global Optimal Solutions in Parametric Problems of Nonlinear Programming, Part I and II. Seminarbericht Nr. 75, Sektion Mathematik, Humboldt-Universität Berlin (1985)

17. Robinson, S.M.: Stability theorems for systems of inequalities. Part II: differentiable nonlinear systems. SIAM J. Numer. Anal. 13, 497-513 (1976)

18. Klatte, D., Li, W.: Asymptotic constraint qualifications and global error bounds for convex inequalities. Math. Program. 84, 137-160 (1999)

19. Klatte, D., Kruger, A., Kummer, B.: From convergence principles to stability and optimality conditions. J. Convex Anal. 19, 1043-1072 (2012)

20. Robinson, S.M.: Regularity and stability for convex multivalued functions. Math. Oper. Res. 1, 130-143 (1976)

21. Cánovas, M.J., Dontchev, A.L., López, M.A., Parra, J.: Metric regularity of semi-infinite constraint systems. Math. Program. B 104, 329-346 (2005)

22. Cánovas, M.J., Klatte, D., López, M.A., Parra, J.: Metric regularity in convex semi-infinite optimization under canonical perturbations. SIAM J. Optim. 18, 717-732 (2007) 